\title{
Research on Garden-style Ecological Outdoor Leisure Sports Industry Clusters in Chengdu
}

\author{
Ling $\mathrm{Su}^{1, \mathrm{a}}$ \\ ${ }^{1}$ Chengdu Medical College, Chengdu, Sichuan Province, China \\ a916556008@qq.com
}

Keywords: Chengdu; Garden-style ecology; Outdoor sports; Leisure industry.

\begin{abstract}
Nowadays, China's social economy develops rapidly, economic structure transforms and industrial structure upgrades. As a result, leisure sports industry faces the opportunity of stepping into a rapid development period. In the process of turning Chengdu into an "Oriental Capital of Leisure", garden-style ecological outdoor leisure sports industry has become more and more important. This article analyzes the garden-style ecological outdoor leisure sports industry clusters in Chengdu from four perspectives: its emergence and development, the characteristics of outdoor leisure sports industry, the significance and development strategies of this industry. Through the study of garden-style ecological outdoor leisure sports industry clusters in Chengdu, not only can we promote the economic transformation and upgrading in this area, but also can provide the important theoretical foundation of speeding up the development of leisure sports industry.
\end{abstract}

\section{The Emergence and Development of Garden-style Ecological Outdoor Sports Industry Clusters in Chengdu}

Chengdu is a central city in west China. It serves as the center of economy, science and technology, cultural and innovation, and external communication. Chengdu is also an integrated transport hub. In recent years, people in Chengdu have fully realized the positive influence and economic benefits of leisure activities. In their spare time, people often take part in outdoor activities like bowling ball games, rafting, mountain climbing, rock climbing, orienteering, ecological wetland viewing and agricultural harvest. These activities help citizens to release their pressure and cultivate their mind, as well as to establish scientific, reasonable ideas of universal leisure and leisure consumption. With the advantages of convenient transportation and developed information system, hundreds of outdoor events and leisure activities for entertaining or public service are held in Chengdu every year, aims to promote the development and prosperity of tourism market.

This part is divided into two sections. The first section, analysis on market environment of outdoor sports industry in Chengdu, goes as follows.

Geographical Conditions. Chengdu located in the hinterland of Chengdu Plain, which lies in the western part of Sichuan Basin. Residents in Chengdu enjoy the flat terrain, crisscrossed river network and abundant natural resources. Agriculture here is developed. Since ancient times Chengdu has been known as "the Land of Abundance". Chengdu is adjacent to Deyang to the east, borders Ya'an to the west and connects Meishan to the south. Chengdu governs ten districts including Jinjiang district, five counties and four county-level cities. Chengdu has a long history and splendid culture. Its name lists in the first National Historical and Cultural Cities and the Best Tour City of China. Chengdu is the starting point of southern Silk Road. More than 2600 years' history of the city enables people here to build many places of interest such as Dujiangyan Dam, Wuhou Temple, Dufu Thatched Cottage and Jinsha Ruins.

Economic Conditions. Chengdu is a national important base of commodity grain and oil, fruits and vegetables, and medicinal herbs. Industry and the tertiary industry in Chengdu develops rapidly. The number of business complexes in Chengdu ranks first in China, while the area of shopping center occupies the second place in the world. By June 2015, 268 of the world top 500 enterprises start their business in Chengdu. Chengdu also owns folk custom clusters like Kuan \& Zhai Alley and Qintai Road, food clusters like Wanda Plaza in Jinhua Road, and business clusters like Songxian Bridge 
Antique Market. The rapid development of economy promotes the improvement of people's living standards, per capital gross domestic product of Chengdu reaches more than 70000 Yuan in 2016, and people have more leisure time to enjoy the fun of outdoor activities. These activities not only expand their social connections, but also improve their quality of life.

Traffic Conditions. Transportation in Chengdu is quite convenient. Urban areas of Chengdu are equipped with several expressways like Jingkun, Hurong, Xiarong, Yurong and Chengli. Main urban zone in Chengdu has 13 bus stations including the Chengdu Shisanling Tomb. Chengdu Station is the largest railway hub in southwest China; it is also the biggest first-class station directly under the control of Chengdu railway administration. Chengdu Station provides trains directly link the capital cities of each province and every municipality, which are directly under the administration of central government. Chengdu Central Station, together with Chengdu West Station, Chengdu South Station, Chengdu East Station and Chengdu Shuangliu International Airport, constitutes a three-dimensional transportation network and a integrated transportation hub in southwest China. This transportation network extending in all directions offers people great convenience to take part in outdoor leisure sports.

The second section, analysis on product demands and consumption structure of outdoor leisure sports market in Chengdu, are listed below.

Market demands on outdoor sports products. Outdoor sports, in which physical exercise occupies the dominant position, have been widely spread in Chengdu. Outdoor sports are moving to the direction of recreation and entertainment. People now tend to moderately relax themselves through taking these activities. In some remote areas, however, square dancing, badminton, shuttlecock and mountain climbing are still the main kinds of outdoor sports. Through related investigations and analyses we found that, the percentage of people who take part in outdoor activities increases in young citizens, middle-aged and elderly residents, as well as people with steady income. This result suggests that nowadays, outdoor sports have been accepted by common families, and today's people are willing to use their leisure time to relax and relieve stress. Accompanied by the increase of participation rate, the demands of outdoor sports products in Chengdu also rise. These increases not only drive the economic transformation and upgrading, but also help people to create new products and service, and gradually form a complete industry chain.

Analysis on outdoor sports consumption structure in Chengdu. There are a plenty of large, comprehensive shopping malls, sports brand shops and individual leisure clothing stores in Chengdu. According to statistics, leisure sports products account for $36 \%$ in the total sales volume of shopping malls. The development of e-commerce platform such as Wechat business, Taobao and Tmall also offers more choices to sports lovers. After investigations we found, in Chengdu, consumers who participate in outdoor sports are fond of Nike, Adidas and other international well-known brands, and are less concern about domestic brands. When making an outdoor sports product, the requirements for its materials, design and processing are strict. Products from foreign brands are expensive, and belong to high-end consumption. Therefore, most people choose domestic products which have beautiful designs and reasonable price. At the same time, the development of tourism and service industry effectively promotes the development of outdoor sports and the related industrial chain in Chengdu.

\section{Characteristics of Garden-style Ecological Outdoor Leisure Sports Industry in Chengdu}

Actively advocating the concept of "Ecological Development". Different from the old thinking, in which designers emphasize beautification over ecological construction, ecological landscape designers pays more attention to the ecological balance and functional benefits, while the emphasis on beautification still remains. It aims to solve the multiple complex contradictions and seek the final balance between urban environment and the ecology, to provide better effect on city greening and to offer more humanized service to the city. Urban ecological greening is an inevitable trend of city development. In the urban ecological greening practice, we need clear plans and schemes, such as the distribution of green plants in different areas, the plans and design on management patterns and target for different biological communities. 
Landscape garden development is the basic approach. The most notable feature of landscape garden is the natural, comfortable environment for "green living". The modern garden culture deepens this connotation. It is mainly manifested in the "people-oriented" philosophy. Modern natural landscape gardens are built on the basis of "beauty originates from nature" concept. That is, when building gardens, people should respect the local ecology, and change only a little on the original environment; building materials should be mainly come from local natural materials. Only in this way, can landscape gardens fully show the natural characteristics of specific regions when they serve the society. Through the building of landscape gardens, outdoor leisure sports can take place in a more natural environment. Ecological garden also plays a role in education, since people can feel and understand the nature better in these gardens.

Tourism development is lead by leisure sports industry. The most important function of playing sports is to build a strong body, while tourists can help people to relax and have fun. The combination of sports and tourist can take the advantages of doing sports, and helps people to enjoy the exchanges and edification of culture, entertainment, leisure, contests and rehabilitation. Sports tourism is a new intersection industry which combines sports and tourism. It can not only brings a great rise to national economy, but also plays a huge role on social service. It is also a part of people's well-being project. It directly benefit ordinary citizens, thus the development of sports tourism is directly related to the improvement of people's living standard, as well as the uplifting of their spiritual and cultural level.

\section{Significance of Developing Garden-style Ecological Outdoor Leisure Sports Industry in Chengdu}

Outdoor leisure sports industry has gradually become a new economic growth point in Chengdu. Chengdu outdoor leisure sports industry has entered a rapid development period since 2008. Its profits reached 11.252 billion Yuan in 2010, and increased by 0.53 times compared with 2008. The growth value reached 2.362 billion Yuan, which is almost 1.7 times of 2008. In 2008, outdoor leisure sports industry accounted for $0.35 \%$ in Chengdu's gross domestic product (GDP). It is an important phenomenon in industry growth. Now, the number of employees in outdoor leisure sports industry has reached nearly 50 thousand. Service projects like gymnasium service, fitness and leisure sports service and intermediary service, have achieved nearly 300 thousand Yuan income, which is more than two times of 2008. The whole picture of outdoor leisure sports industry shows that a stable system has established in Chengdu.

Outdoor leisure sports industry is a "sunrise industry" with low energy consumption and high added value. In recent years, the rapid economic development in Chengdu has gradually changed residents' consumption habits. Citizens still work hard, but at the same time they begin to care about the physical conditions of themselves and their family members. Therefore, consumption ratio of outdoor leisure sports increased. Outdoor leisure sports is an industry with little pollution and high productive rate, and it develops along with the social development. It has become an essential force that influences people's consumption concepts. At present, Chengdu outdoor leisure sports industry has become the best in western regions of China, and has laid a solid foundation for the development of other projects.

Outdoor leisure sports industry promotes the industrial structure adjustment and economic development. Speaking of the industrial structure of outdoor leisure sports in Chengdu, sports equipment, sports wear and sports field are the main sources of income. However, sports competition, which serves as the main body of outdoor leisure sports industry, does not takes a high proportion in the income volume. It does not reach the effect of promoting the industry. The effect of industrial development is often affected by the industrial structure. Outdoor leisure sports can not only drive the development of other industries in Chengdu, but also promote Chengdu citizens' overall consumption level from "survival" to "enjoy". Thus, Chengdu government should formulate the construction goal and development idea of building a "modern garden city", and take the path of internationalization development. The concept of lifelong sports should also be strengthened. In this way, outdoor leisure industry can drive the economic development and, at the same time, enhance residents' awareness of 
playing sports and improving their physical quality. The prosperity of this industry can also helps our country to become a sports power.

\section{Strategies of Developing Outdoor Leisure Sports Industry Clusters in Chengdu}

Outdoor sports play the fundamental role, and tourism promotes the development of outdoor sports. During the development process of garden-style ecological outdoor leisure sports industry in Chengdu, we need to adhere to ecological concept constantly, and accelerate the construction of public service system and recreational industry. We need to make full use of the functions of outdoor sports production, and to create products which can satisfy people's demands on outdoor leisure sports. Organizing a series of outdoor activities such as skiing, drifting and golf can broaden the development space of leisure sports, while providing a large number of outdoor sports products can meet people's demands. To create a favorable environment of outdoor sports development, we ought to optimize the public service, foster the third industry, improve the service quality and efficiency, and strengthen the supervision and management. Actively promote the development of leisure industry is an important way to improve productivity, and to promote the development of leisure tourism industry.

Reasonable programming and brand management can raise products' quality and production efficiency. Chengdu garden-like ecological outdoor leisure sports industry is still in its primary stage of development. The industry is lack of dominant, leading enterprises and famous brands. These adverse factors lead to the small scale of outdoor leisure industry and make local brands less competitive when facing foreign ones. In recent years, foreign brands in outdoor sports industry develop very fast. In order to enhance their international competitiveness and win in this fierce international competition, outdoor sports industry in Chengdu should make technological innovation, develop local brands, improve industrial manufacturing capabilities and try to realize scale management system. The cooperation between scientific research institutions, universities and local brands such as Jinlang, Feiyang and Wannianqing should be strengthened. They need to constantly develop new products and technology, and target their consumers in middle class citizens, then try to build up famous brands.

Integrating resources and extending industrial chain, aiming to achieve properly centralized development. In general, the scale of outdoor sports in Chengdu is still very small and the development of outdoor leisure sports industry is still in its infancy. The distribution is uneven, and most of these activities are concentrated in urban areas. The scale of outdoor sports is not big enough to build an independent industry. Therefore, it is important to accelerate the creation of corporate structure and mechanism. The authority needs to reinforce the supervision for outdoor sports projects. Scientific plans, relevant supporting policies and feasible measures should also be made. The ideal market operation pattern should be concentrated, market-based, social-participated, and be leaded by the government. The industry needs to integrate related resources, service and management. Starting with the current situation, outdoor sports in Chengdu is ought to be promoted to achieve healthy and sustainable development.

Reflecting local features of Chengdu culture, and promoting the development of outdoor leisure industry. To better promote the development of Chengdu garden-like outdoor sports, we need to fully understand the natural resources in Chengdu, as well as the cultural connotation and characteristics of leisure industry in this city. The unique charm of Chengdu should be reflected from multiple perspectives, and Chengdu's reputation of "leisure city" needs to be activated constantly. The development of outdoor leisure industry should be hinged around historical sites and humanity landscapes like Wuhou Temple, Dufu Thatched Cottage, Yongling Mausoleum, Qingyang Palace, Wangjiang Mansion, Wenshu Monastery, Mingshuwang Tomb and Zhaojue Temple in Chengdu. The "Chengdu Top Ten Scenes; New Top Ten Scenes" can also be served as carriers to show the features of Chengdu outdoor products. With the development of leisure industry clusters, they can form unique cultural features, artistic charm and enduring vitality, which in turn can promote the healthy and sustainable development of outdoor leisure industry. 
To sum up, the development of the Chengdu garden-like ecological outdoor leisure sports industry clusters reflects the features of Chengdu outdoor leisure industry. It promotes the economic transformation and upgrading in Chengdu area, provides new powers to speed up the development of leisure sports industry, and promotes Chengdu economy to develop steadily.

\section{Acknowledgement}

Fund project: 2014 Key Research Foundation on Humanities and Social Sciences (Leisure Sports Industry) for Youth Projects of Education Department of Sichuan Province (Project No.: XXTYCY2014C04).

\section{References}

[1] WANG Jin. Research on the Development Situation and Strategies of China's Outdoor Sports [J]. Market Modernization, 2008, 550.

[2] LI Hong-yan. Research on Theories and Practice of Outdoor Sports [J]. Journal of Beijing University of Physical Education, 2006 (4).

[3] LI Jiu-quan. Research on the Development Situation and Strategies of China's Outdoor Sports [J]. Journal of Beijing University of Physical Education, 2008 (12). 